\title{
Relação entre variáveis climáticas e a mortalidade de idosos por doenças cardiovasculares
}

\author{
Natália Galvão*, Maysa de Lima Leite**
}

\section{Resumo}

O estudo objetiva avaliar a relação entre a mortalidade por doenças do aparelho cardiovascular (DACs) e variáveis climáticas, assim como descrever os óbitos de idosos no município de Ponta Grossa, Paraná, em um período de 16 anos (1998-2013). Os dados epidemiológicos foram analisados em formato de coeficiente de mortalidade mensal juntamente com os dados climáticos pelo teste de coeficiente de correlação de Pearson e por regressão linear múltipla. As DACs foram a maior causa de mortalidade na população idosa do município investigado. O coeficiente de mortalidade apresentou um comportamento sazonal pouco evidente durante a série histórica. Foram observadas fortes correlações negativas com as temperaturas que apresentaram alta significância estatística. Os modelos regressivos anuais apresentaram alta significância e um bom grau de ajuste. É possível comprovar que as variáveis climáticas exercem influência na mortalidade por doenças cardiovasculares no município de Ponta Grossa, destacando a influência da temperatura.

Palavras-chave: Climatologia. Doenças cardiovasculares. Epidemiologia. Idoso. Mortalidade de idosos.

\section{Introdução}

As doenças crônicas passaram a determinar a maioria das causas de óbitos no Brasil, seguindo a tendência mundial, modificando o perfil das doenças que ocorrem na população. Tais doenças, que incluem as doenças do aparelho circulatório (DACs), atingem de forma mais intensa aqueles pertencentes aos grupos vulneráveis, como os idosos (PIUVEZAM et al., 2015).

Apesar de as DACs serem a principal causa de mortalidade no Brasil, assim como no mundo, existem poucos estudos que abordam a distribuição temporal da mortalidade por elas, principalmente no que se refere à população idosa (PIUVEZAM, et al., 2015).

Os idosos apresentam maior carga de doenças que o restante da população, por isso, necessitam utilizar mais os serviços de saúde. O maior problema dos modelos

* Bacharel em Enfermagem. Mestranda em Ciências da Saúde na Universidade Estadual de Ponta Grossa. Enfermeira na Santa Casa de Irati. Endereço: Urbano Messias, 100, Irati, PR, CEP: 84500-000. E-mail: gn_natalia@hotmail.com

** Graduada em Agronomia. Doutora em Agronomia. Professora do Departamento de Biologia Geral da Universidade Estadual de Ponta Grossa. E-mail: mleite@uepg.br

$\rightarrow$ http://dx.doi.org/10.5335/rbceh.v14i3.6861

Recebido em: 28.03.2017. Aceito em: 03.04.2018 
assistenciais vigentes é o foco exclusivo na doença, deixando de fora a preocupação com fatores de risco e determinantes ambientais (VERAS, 2011, 2012).

Com o envelhecimento, as pessoas passam, gradativamente, a apresentar condições fisiológicas e/ou fisiopatológicas diferentes, com necessidades e características próprias da faixa etária, o que requer estudos direcionados para essa parcela da população.

É importante destacar que há determinantes ambientais da saúde humana, como o clima, que influencia o ritmo biológico, interfere em todas as atividades e funções humanas, evidenciando que os seres humanos têm uma variabilidade adaptativa individual, o que interfere mais ou menos em sua sensibilidade ao clima e, dessa forma, em seu conforto e saúde (NOGUEIRA et al., 2011).

Os idosos são a população mais acometida por diversas doenças, pois são o grupo etário com maior vulnerabilidade. Nesse sentido, conclui-se que os idosos também apresentam maior "climatosensibilidade", ou seja, as variações climáticas são sentidas mais intensamente nessa faixa etária (GENARO, 2011).

A associação entre o clima e a saúde humana precisa ser definida, em razão da necessidade de desenvolvimento de medidas de mitigação pelos agentes de saúde pública, assim como da adoção dessas medidas pela população de risco, visto que o envelhecimento da população tem amplitude mundial.

A mortalidade é utilizada como referência para a avaliação de saúde como um todo ou subdividida em estratos populacionais, como no caso do presente estudo, que investiga apenas os idosos (JORGE et al., 2008). A análise da mortalidade contribui para a definição das prioridades de atuação dos serviços de saúde na prevenção e promoção, atuando nos fatores determinantes para as doenças e organizando o serviço de acordo com a demanda local. Nessa perspectiva, é de fundamental importância estabelecer e antecipar a periodicidade e sazonalidade das condições mais incidentes em cada município, permitindo assim o planejamento para o enfrentamento de tais problemas (MIRANDA, 2009).

Na perspectiva da influência de fatores ambientais, como o clima, na saúde do idoso, verifica-se a necessidade de conhecer a relação entre a mortalidade por doenças cardiovasculares e o clima. $\mathrm{O}$ conhecimento da tendência de mortalidade por doenças cardiovasculares em idosos, população de risco, representa um importante indicador para a compreensão do processo saúde-doença.

Nesse contexto, este trabalho teve como objetivos avaliar a relação entre a mortalidade de idosos por DACs e as variáveis climáticas - temperatura do ar, precipitação pluvial e umidade relativa do ar -, e descrever os óbitos de idosos no município de Ponta Grossa, Paraná, em um período de 16 anos (1998-2013).

\section{Metodologia}

Trata-se de um estudo ecológico, com o intuito de estabelecer relações entre mortalidade por doenças cardiovasculares e variáveis climáticas no período 
de janeiro de 1998 a dezembro de 2013 no município de Ponta Grossa, Paraná. Primeiramente, foi realizado um levantamento dos dados referentes aos óbitos por DACs, por meio do Departamento de Informática do Sistema Único de Saúde (DATASUS), no site do Ministério da Saúde. As DACs correspondem ao Capítulo IX, do Código Nacional de Doenças (CID-10), e nele estão inclusas as doenças nos seguintes agrupamentos: doenças reumáticas do coração (I00-I09); doenças hipertensivas (I10-I15); doenças isquêmicas do coração (I20-I25); doenças cardíacas pulmonares (I26-I28); outras formas de doenças do coração (I30-I52); doenças cerebrovasculares (I60-I69); doenças das artérias, arteríolas e capilares (I70-I79); doenças de veias, vasos e gânglios linfáticos (I80-I89); outros transtornos (I95-I99) (DATASUS, 2016).

As informações das DACs coletadas foram: número de óbitos, faixa etária e causas por categorias, de sujeitos residentes no município de Ponta Grossa. Em seguida, os dados foram organizados e tabulados no Microsoft Exce ${ }^{\circledR}$ e, depois, foram elaborados gráficos de distribuição mensal, sazonal e anual para a análise posterior.

Para o levantamento referente à população da localidade no período de 1998 a 2012, reuniram-se os dados de estimativas, censos e contagem populacional disponibilizados pelo Instituto Brasileiro de Geografia e Estatística no site do DATASUS, classificados por faixa etária. Para o ano de 2013, sem informação, ajustou-se um modelo de regressão linear com um ajuste de $R^{2}=0,9540$. Com a estimativa anual concluída, foi possível fazer a distribuição mensal, o que permitiu a realização da análise sazonal.

Após a conclusão das estimativas mensais, foram realizados os cálculos do coeficiente de mortalidade mensal de DACs por 10.000 habitantes, utilizando a equação 1 :

$C M=\left(\frac{\text { Número de óbitos de idosos por DAC }}{\text { Metade da população de idosos no período }}\right) * 10.000$

\section{Equação 1}

Para o cálculo do coeficiente de mortalidade (CM) por DAC, utilizou-se a média da população central (junho e julho) de cada ano. Sendo assim, o número de casos de cada mês foi divido pela média da população idosa central do seu respectivo ano e, na sequência, multiplicado por 10.000 habitantes (REDE INTERAGENCIAL DE INFORMAÇÃO PARA A SAÚDE, 2008)

Os registros climáticos diários, para o período de 1998 a 2013, foram gerados a partir do "PGECLIMA_R: Gerador Estocástico de Cenários Climáticos", que forneceu uma simulação das variáveis: precipitação pluviométrica, umidade relativa do ar, temperaturas máxima e mínima, tomando como base de dados a série histórica (1980 a 2009) da Estação Meteorológica pertencente ao Instituto Agronômico do Paraná (Iapar), com coordenadas geográficas de $25^{\circ} 13^{\prime}$ para latitude sul, longitude $50 \square 01$ ' Oeste e altitude de 880 metros. Os dados foram então organizados em planilhas do Microsoft Excel ${ }^{\circledR}$ e utilizados para o 
desenvolvimento de gráficos, tabelas e demais análises.

Os coeficientes de mortalidade das médias mensais juntamente com as médias mensais das variáveis climáticas, precipitação pluviométrica, umidade relativa e temperaturas máximas, mínimas e médias, foram analisadas por meio da estimativa do coeficiente de correlação de Pearson $(r)$, o qual mensura o grau de relação mútua entre duas variáveis. Para avaliação da significância dos coeficientes de correlação obtidos, utilizou-se $o$ teste $t$ de Student.

Os coeficientes de mortalidade também foram avaliados conjuntamente com os dados referentes às variáveis climáticas para a análise de possíveis interferências do clima. Para tanto, realizou-se a análise de modelos matemáticos anuais e sazonais de regressão linear múltipla para verificar a influência dessas variáveis sobre os coeficientes de mortalidade. O modelo teórico em questão (DOWNING; CLARK, 2003) pode ser representado pela equação 2 :

$$
Y_{i}=\beta_{0}+\beta_{i 1} X_{i 1}+\beta_{i 2} X_{i 2}+\beta_{i 3} X_{i 3}+\epsilon_{i}
$$

Equação 2

Em que: Y é a variável explicada ou dependente (coeficiente de mortalidade), $\beta 0, \beta 1, \beta 2$ e $\beta 3$ são os parâmetros desconhecidos; Xi1, Xi2 e Xi3 são as variáveis independentes (variáveis climáticas); e $\epsilon_{\mathrm{i}}$ é o erro residual.

Concomitantemente à elaboração dos modelos matemáticos mensais, realizou-se o cálculo dos coeficientes de determinação ( $\mathrm{R}^{2}$ múltiplo) para cada modelo juntamente com seu valor de $p$, para analisá-los quanto às suas capacidades de justificar os coeficientes de mortalidade a partir das variáveis climáticas e admitir qual a sua significância estatística.

O estudo utilizou apenas dados secundários, sem identificação dos sujeitos, e foi realizado em conformidade com os princípios éticos da Resolução do Conselho Nacional de Saúde (CNS) no ${ }^{\circ} 466$, de 12 de dezembro de 2012.

\section{Resultados}

Entre os anos de 1998 a 2013, o município de Ponta Grossa, PR, apresentou um total de 31.569 óbitos, sendo que $7.708(24,42 \%)$ casos foram pelas DACs; e $5.937(77,02 \%)$ casos de DACs foram em idosos. Assim, é possível concluir que as DACs foram a maior causa de mortalidade na população idosa de Ponta Grossa. Conforme a Figura 1, a segunda maior causa de óbitos seria por neoplasias, seguidas por doenças do aparelho respiratório, doenças endócrinas, nutricionais e metabólicas e doenças do aparelho digestivo.

As DACs apresentam-se, continuamente, como a maior causa de mortalidade dentro da série histórica. As cinco maiores causas de mortalidade no município encontram-se em um padrão de crescimento do número de casos ao longo dos anos, sugerindo, assim, que a mortalidade de idosos no município encontra-se em pleno crescimento. Verifica-se que as DACs levaram a um 
número de óbitos muito maior quando comparadas às outras causas, sendo que estas ainda demonstram uma pequena variação entre a mortalidade, fazendo com que as linhas se encontrem em determinados anos, como é o caso mais evidente das doenças endócrinas, nutricionais e metabólicas e do aparelho digestivo (Figura 1).

Figura 1 - Principais causas de mortalidade na população idosa de Ponta Grossa, Paraná, no período de 1998 a 2013

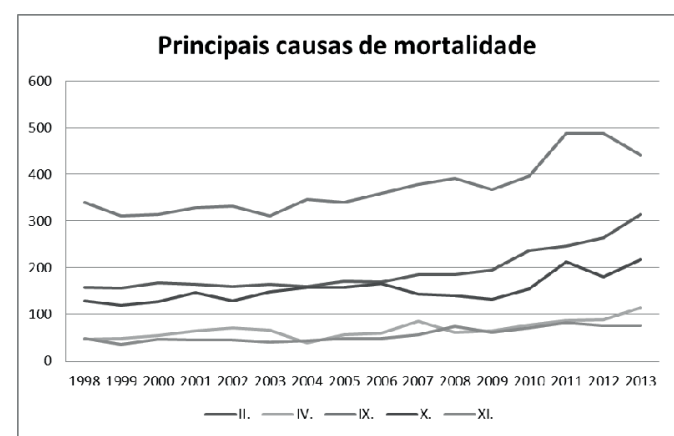

Fonte: elaboração das autoras.

Legenda: II. Neoplasias; IV Doenças endócrinas, nutricionais e metabólicas; IX. Doenças do aparelho circulatório; X. Doenças do aparelho respiratório; XI. Doenças do aparelho digestivo.

Os idosos são os mais acometidos pela mortalidade por DACs no município de Ponta Grossa, PR (Figura 2). Observa-se, ainda, que o número de óbitos aumentou com o acréscimo da idade. Para o município de Ponta Grossa, a faixa etária em que mais ocorreram óbitos foi a de 70 a 79 anos.
Figura 2 - Mortalidade por DACs por faixa etária, Ponta Grossa, PR, no período de 1998 a 2013

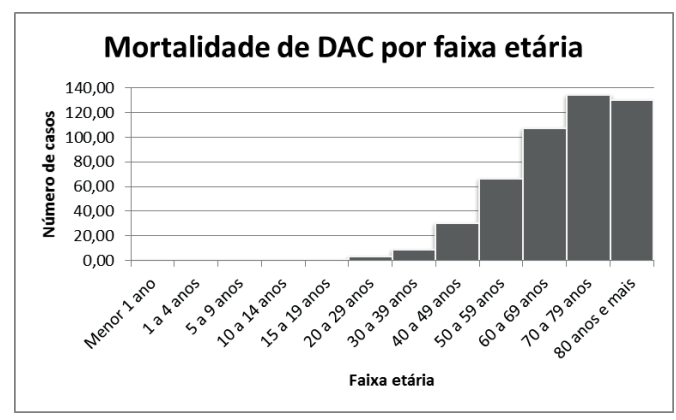

Fonte: elaboração das autoras.

Dentro das doenças cardiovasculares, o grupo de achaques que mais acometeu a população de idosos foi o grupo das doenças cerebrovasculares, com 36\% dos óbitos (2.136); em segundo lugar, as doenças isquêmicas do coração, com 26\% dos óbitos (1.545); em terceiro, outras doenças do coração, com $22 \%$ dos óbitos (1.290). As doenças hipertensivas do coração também apresentaram quantidade de óbitos significativa $(8 \%-487)$.

O coeficiente de mortalidade (Figura 3) foi utilizado para caracterizar a distribuição mensal e sazonal dos óbitos e discriminar tais características em cada faixa etária da população idosa. 
Figura 3 - Coeficientes de mortalidade de idosos segundo faixa etária, para cada 10.000 habitantes, Ponta Grossa, PR, de 1998 a 2013

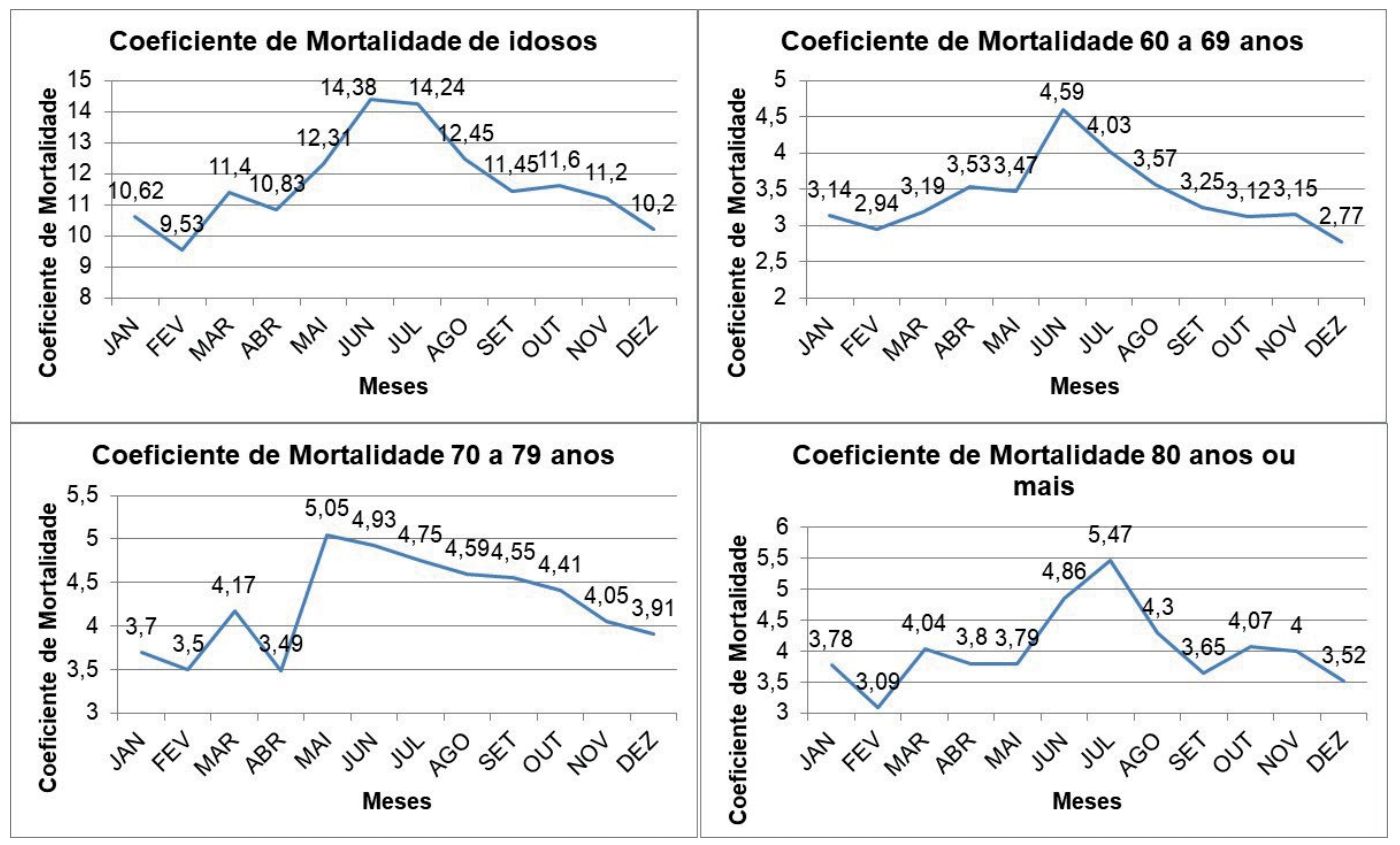

Fonte: elaboração das autoras.

As DACs apresentam um comportamento sazonal pouco evidente, no qual apresentaram, nos primeiros meses do ano, um baixo coeficiente de mortalidade para todas as faixas etárias, mas que se eleva no meio do ano e, em seguida, decai, mantendo-se mais baixo no fim do ano. Nota-se, no entanto, que, em todas as faixas etárias, há picos de mortalidade nos meses em que a atmosfera apresenta uma variabilidade maior de temperatura (maio, junho e julho), nos quais ela passa a apresentar temperaturas mais baixas. Tais picos fazem com que o coeficiente de mortalidade dos idosos mantenha-se elevado nesses mesmos meses. Há, ainda, uma tendência de deslocamento dos picos de mortalidade para a direita (Figura 3).
Em cada faixa etária, o coeficiente de mortalidade tem um padrão específico, sugerindo que as DACs agem de formas diferentes em cada indivíduo. $\mathrm{O}$ coeficiente de mortalidade da faixa etária de 60 a 69 anos apresenta-se com valores mais baixos durante os meses quentes e eleva-se à medida que as temperaturas decrescem nos meses frios, com pico em junho, e após decai. No coeficiente de 70 a 79 anos, existem elevações e decréscimos durante os quatro primeiros meses do ano, e seu pico ocorre em maio, com decréscimo lento até os últimos meses do ano.

Os coeficientes de mortalidade da faixa etária de 80 anos ou mais e a de idosos são similares em suas curvas, 
apresentando elevações e decréscimos do coeficiente. A diferença entre os coeficientes de um mês para o outro é pequena, dificultando assim a identificação das possíveis interações com o ambiente.

Verificaram-se fortes correlações negativas com as temperaturas máximas, médias e mínimas, com alta significância estatística $(p)$. Para todas as faixas etá- rias, os valores do coeficiente de correlação de Pearson para as temperaturas foram próximos, e, nas diferentes faixas etárias, os melhores resultados foram obtidos pelas temperaturas. A precipitação pluvial e a umidade relativa do ar não apresentaram correlações com significância estatística em nenhuma faixa etária (Tabela 1).

Tabela 1 - Correlações entre os coeficientes de mortalidade e as variáveis climáticas

\begin{tabular}{|c|c|c|c|c|c|}
\hline Idosos & & $r$ (Pearson) & $\mathrm{R} 2$ & Test T & (p) \\
\hline & Precipitação pluvial & $-0,4895$ & 0,2396 & $-17,752$ & 0,1062 \\
\hline & Temperatura Máxima & $-0,8839$ & 0,7813 & $-59,775$ & 0,0001 \\
\hline & Temperatura média & $-0,8879$ & 0,7883 & $-6,103$ & 0,0001 \\
\hline & Temperatura mínima & $-0,8892$ & 0,7906 & $-61,454$ & 0,0001 \\
\hline & Umidade relativa & $-0,0501$ & 0,0025 & $-0,1586$ & 0,8771 \\
\hline \multirow[t]{6}{*}{60 a 69 anos } & & r (Pearson) & $\mathrm{R} 2$ & Test $\mathrm{T}$ & (p) \\
\hline & Precipitação pluvial & $-0,5001$ & 0,2501 & $-18,264$ & 0,0977 \\
\hline & Temperatura Máxima & $-0,8142$ & 0,6629 & $-44,349$ & 0,0013 \\
\hline & Temperatura média & $-0,8028$ & 0,6445 & $-42,577$ & 0,0017 \\
\hline & Temperatura mínima & $-0,7905$ & 0,6249 & $-40,813$ & 0,0022 \\
\hline & Umidade relativa & 0,1710 & 0,0293 & 0,5489 & 0,5951 \\
\hline \multirow[t]{6}{*}{70 a 79 anos } & & $r$ (Pearson) & $\mathrm{R} 2$ & Test $\mathrm{T}$ & (p) \\
\hline & Precipitação pluvial & $-0,3264$ & 0,1065 & $-10,918$ & 0,3005 \\
\hline & Temperatura Máxima & $-0,8543$ & 0,7298 & $-51,973$ & 0,0004 \\
\hline & Temperatura média & $-0,8586$ & 0,7371 & $-52,954$ & 0,0003 \\
\hline & Temperatura mínima & $-0,8577$ & 0,7356 & $-52,745$ & 0,0004 \\
\hline & Umidade relativa & $-0,1019$ & 0,0104 & $-0,3238$ & 0,7528 \\
\hline \multirow[t]{6}{*}{80 anos ou mais } & & $r$ (Pearson) & $\mathrm{R} 2$ & Test T & (p) \\
\hline & Precipitação pluvial & $-0,4746$ & 0,2253 & $-17,053$ & 0,1189 \\
\hline & Temperatura Máxima & $-0,6995$ & 0,4893 & $-30,954$ & 0,0113 \\
\hline & Temperatura média & $-0,7145$ & 0,5106 & $-32,297$ & 0,009 \\
\hline & Temperatura mínima & $-0,7283$ & 0,5305 & $-33,612$ & 0,0072 \\
\hline & Umidade relativa & $-0,1707$ & 0,0291 & $-0,5478$ & 0,5958 \\
\hline
\end{tabular}

Fonte: elaboração das autoras. 
A maioria das correlações apresentou o sinal negativo, indicando que, à medida que a variável sofre decréscimos, o coeficiente de mortalidade eleva-se. A única exceção foi o coeficiente de mortalidade de 60 a 69 anos com a umidade relativa, que apresentou sinal positivo em seu resultado, sugerindo que o coeficiente de mortalidade se eleva quando há a elevação da umidade, apesar de que esta correlação não obteve significância estatística $(p=0,5951)$.

Considerando que as temperaturas nas correlações se comportaram de modos diferentes, porém próximos, em cada faixa etária, optou-se por utilizar as temperaturas médias juntamente com a precipitação pluvial e a umidade relativa do ar para se calcular as regressões (Tabela 2).

Tabela 2 - Modelos sazonais e anuais de regressões lineares múltiplas e seus respectivos coeficientes de regressão $\left(\mathrm{R}^{2}\right)$ e $p$ value $(\mathrm{p})$ para a estimativa do coeficiente de mortalidade IDOSO

\begin{tabular}{|c|c|c|c|}
\hline & Modelo da Regressão & R2 & (p) \\
\hline Verão & $\mathrm{CM}=+1,2979+0,0615^{*} \mathrm{PP}+7,191 * \mathrm{~T}$ média $-102,56 *$ UR & 0,2450 & 0,1519 \\
\hline Outono & $C M=+0,5144-0,0261 * P P-1,1875 * T$ média $-30,3486 *$ UR & 0,1139 & 0,5018 \\
\hline Inverno & $C M=+0,8469-0,0017 * P P+3,0618 * T$ média $-30,5956 *$ UR & 0,1747 & 0,7030 \\
\hline Primavera & $C M=+0,6479+0,0128 * P P+2,2058 * T$ média $-91,9244 * U R$ & 0,1394 & 0,5938 \\
\hline Anual & $\mathrm{CM}=+10,0232-0,0007^{*}$ PP $-0,4277^{*}$ T média $+2,266^{*}$ UR & 0,7899 & 0,0012 \\
\hline \multicolumn{4}{|l|}{60 A 69 ANOS } \\
\hline & Modelo da Regressão & R2 & (p) \\
\hline Verão & $\mathrm{CM}=+2,0559+0,0219 * \mathrm{PP}+0,6776 *$ T média $+14,0844 * \mathrm{UR}$ & 0,3395 & 0,0554 \\
\hline Outono & $\mathrm{CM}=+0,7031-0,0149 * \mathrm{PP}-0,5528 * T$ média $+10,3427 *$ UR & 0,1495 & 0,6279 \\
\hline Inverno & $\mathrm{CM}=+0,6032+0,0156 * \mathrm{PP}+1,6983 * \mathrm{~T}$ média $-17,3585 *$ UR & 0,1310 & 0,5639 \\
\hline Primavera & $\mathrm{CM}=+0,2558+0,011 * \mathrm{PP}+0,0551 * \mathrm{~T}$ média $-6,5099 * \mathrm{UR}$ & 0,0601 & 0,7474 \\
\hline Anual & $\mathrm{CM}=+7,4534-0,0033^{*}$ PP $-0,117 *$ T média $+5,8601 *$ UR & 0,7365 & 0,0026 \\
\hline \multicolumn{4}{|l|}{70 A 79 ANOS } \\
\hline & Modelo da Regressão & R2 & (p) \\
\hline Verão & $\mathrm{CM}=+1,0724+0,0242 * \mathrm{PP}+2,7533^{*} \mathrm{~T}$ média $-68,1877^{*}$ UR & 0,2114 & 0,2109 \\
\hline Outono & $\mathrm{CM}=+0,3996-0,0073 * \mathrm{PP}-0,322 *$ T média $+31,509 *$ UR & 0,0908 & 0,6011 \\
\hline Inverno & $\mathrm{CM}=+0,7404-0,0114 * \mathrm{PP}+1,609 * \mathrm{~T}$ média $-6,3801 * \mathrm{UR}$ & 0,1562 & 0,6492 \\
\hline Primavera & $\mathrm{CM}=+0,2796-0,0063 * \mathrm{PP}+0,5032 * \mathrm{~T}$ média $+34,6135 * \mathrm{UR}$ & 0,0653 & 0,7220 \\
\hline Anual & $\mathrm{CM}=+9,3013+0,0042 * \mathrm{PP}-0,1754 *$ T média $-1,9111 *$ UR & 0,7772 & 0,0015 \\
\hline \multicolumn{4}{|l|}{80 ANOS OU MAIS } \\
\hline & Modelo da Regressão & R2 & (p) \\
\hline Verão & $\mathrm{CM}=+0,5669+0,0153 * \mathrm{PP}+3,7404 * \mathrm{~T}$ média $-48,2083 *$ UR & 0,1241 & 0,5380 \\
\hline Outono & $\mathrm{CM}=+1,4423-0,004 * \mathrm{PP}-0,296 *$ T média $-72,1911 *$ UR & 0,2650 & 0,1240 \\
\hline Inverno & $\mathrm{CM}=+0,0638-0,0059 * \mathrm{PP}-0,2371 * \mathrm{~T}$ média $-6,8736 * \mathrm{UR}$ & 0,0157 & 0,9557 \\
\hline Primavera & $\mathrm{CM}=+4,414+0,0083 * \mathrm{PP}+1,6504 * \mathrm{~T}$ média $-120,447 * \mathrm{UR}$ & 0,5246 & 0,0054 \\
\hline Anual & $\mathrm{CM}=+2,9432-0,0015 * \mathrm{PP}-0,1361 * \mathrm{~T}$ média $-1,7689 * \mathrm{UR}$ & 0,5246 & 0,0311 \\
\hline
\end{tabular}

Fonte: elaboração das autoras.

Legenda: CM: coeficiente de mortalidade; PP: precipitação pluvial; T média: temperatura média; UR: umidade relativa do ar. 
Os resultados sazonais obtidos apresentaram ajustes fracos, com exceção do período da primavera na faixa etária de 80 anos ou mais, que obteve elevada significância estatística $(\mathrm{p}=0,0054)$ e um ajuste razoável $\left(\mathrm{R}^{2}=0,5246\right)$. Os modelos regressivos anuais, de outro modo, apresentaram alta significância em todas as faixas etárias e um bom grau de ajuste.

\section{Discussão}

O contínuo aumento da mortalidade de idosos demonstrado pelo estudo pode ser atribuído ao aumento da população idosa, em que mais pessoas alcançam idades maiores que 60 anos, e, quando falecem já nessa faixa etária, aumentam o coeficiente de mortalidade de idosos.

Apesar de o processo de envelhecimento não estar, necessariamente, relacionado a doenças e incapacidades, os hábitos de vida influenciam no desenvolvimento de doenças cardiovasculares, que se acumulam no indivíduo idoso (ALVES et al., 2007; FECHINE; TROMPIERI, 2012).

O estudo demonstrou um elevado número de óbitos causados por DACs no município investigado, além de neoplasias e doenças respiratórias, esse resultado foi similar ao encontrado em estudos realizados no estado do Rio Grande do Sul e em Londrina, no Paraná, em que as DACs foram também a principal causa de mortalidade de idosos, seguidas pelas neoplasias e doenças do aparelho respiratório (GOTTLIEB et al., 2011; CABRERA; ANDRADE; WAJNGARTEN, 2007). As doenças cardiovasculares também são a maior causa de mortalidade no Brasil e no mundo (NOGUEIRA et al., 2011; DATASUS, 2016).

A faixa etária mais acometida pela mortalidade por DACs no município de Ponta Grossa, PR, refere-se aos idosos na faixa etária de 70 a 79 anos, enquanto em Londrina, houve um predomínio de óbitos entre os indivíduos de 80 anos ou mais em relação ao grupo com 60 a 69 anos (CABRERA; ANDRADE; WAJNGARTEN, 2007).

Há diferenças entre os idosos com o passar da idade, pois, gradativamente, ocorre um processo em que o organismo começa a apresentar declínio, é a fase inicial da velhice, e, progressivamente, aparecem danos em algumas funções, por fim, têm-se a idade mais avançada, na qual os indivíduos carecem de cuidados especiais.

O presente estudo demonstrou que a população idosa de Ponta Grossa apresentou maior número de mortalidade na faixa etária de 70 a 79 anos, demonstrando que os idosos não conseguem chegar a uma idade mais avançada e morrem com a perda de funções associadas às patologias (FECHINE; TROMPIERI, 2012).

$\mathrm{O}$ aumento da expectativa de vida, que ocorre mundialmente nas últimas décadas, faz com que haja uma concentração da mortalidade durante o período em que os indivíduos já se tornaram idosos, atingido mais de $60 \%$ dos óbitos. Salienta-se, ainda, que há uma mudança gradual no perfil das doenças que são determinantes para a mortalidade do idoso (CABRERA; ANDRADE; WAJNGARTEN, 2007). 
Todas as faixas etárias apresentaram picos de mortalidade nos meses em que a atmosfera apresenta uma variabilidade maior de temperatura (maio, junho e julho), em que ela passa a apresentar temperaturas mais baixas. As ondas de calor e frio, comumente encontradas nesse período de maior variabilidade de temperatura, são de fundamental importância para a ocorrência de óbitos, porque expõem o indivíduo idoso a condições que ele não está acostumado ou que excedem sua capacidade de termorregulação, e uma doença poderá se agravar ou levar ao óbito (SOUZA; SILVA; LACERDA, 2013).

O frio é reconhecido como fator potencial de algumas doenças, entre elas as cardiovasculares, que podem apresentar características de sazonalidade, com acréscimos no período do inverno (PASCOALINO; PITTON, 2012). As temperaturas extremas causam aumento da mortalidade em extremos de idades, como é o caso dos idosos.

A mortalidade por doenças cardiovasculares aumenta com a diminuição das temperaturas, e a diminuição de $1^{\circ} \mathrm{C}$ na temperatura é associada ao aumento de $1,72 \%$ e $1,25 \%$ de mortes por causas cardiovasculares e cerebrovasculares, respectivamente, com predomínio na população idosa (NOGUEIRA et al., 2011).

Nesta pesquisa, foram verificadas fortes correlações negativas com as temperaturas máximas, médias e mínimas. A temperatura máxima foi a mais fortemente correlacionada com a mortalidade por doenças cardiovasculares, isso representa que o impacto associado com tem- peraturas extremas no inverno é muito diferente do que com as temperaturas extremas do verão. As variáveis térmicas constituem um fator de adoecimento e óbito, tanto as temperaturas baixas como as elevadas constituem riscos à saúde (SILVA; RIBEIRO; SANTANA, 2014).

O ser humano encontra-se em um estado de interação permanente com a atmosfera, em que se busca o equilíbrio entre a produção e a perda de calor. Sendo o homem homeotérmico, ele possui um sistema de termorregulação capaz de manter a temperatura corporal constante independente das variações de temperatura do ambiente externo. Esse sistema mantém a temperatura corporal pela produção de calor como subproduto de processos metabólicos (termogênese) ou pela perda de calor para o ambiente externo (termólise) (SILVA; RIBEIRO; SANTANA, 2014). Desse modo, quando o calor do ambiente é maior que o do corpo, há sensação de calor e o sistema trabalha para que ocorra a perda de calor (termólise), sendo acionados os mecanismos de resfriamento. Caso o calor dissipado do corpo seja maior que o do ambiente, há sensação de frio e o sistema trabalha na produção de calor (termogênese), sendo acionados os mecanismos de produção de calor (SILVA; RIBEIRO; SANTANA, 2014).

Assim, visando à geração de calor nos períodos mais frios, o organismo intensifica a atividade muscular, ocasionando uma sobrecarga no coração (NOGUEIRA et al., 2011; GENARO, 2011). Entretanto, com o aumento da temperatura, há uma elevação da elimi- 
nação do suor, que pode levar a uma redução do volume plasmático e à queda da pressão arterial. Além disso, há aumento na viscosidade do sangue, causada pela concentração de hemácias, plaquetas e colesterol. A queda da pressão arterial reduz o fluxo sanguíneo nas arteríolas, e as plaquetas junto com o colesterol facilitam o aparecimento de trombos. Estes se envolvem em ateromas e, pela interrupção do fluxo sanguíneo para os tecidos, causam infarto do miocárdio ou acidente vascular cerebral, conforme o órgão afetado (KEATINGE, 1986 apud RUMEL et al., 1993).

Um agravante da condição dos idosos em relação às DACs são as alterações biológicas que ocorrem com o envelhecimento. Há uma diminuição da força de contração e do número de batimentos cardíacos, assim como redução da frequência cardíaca em repouso, aumento do colesterol e da resistência vascular periférica, com o consequente aumento da pressão sanguínea, condições estas que estão envolvidas em processos patológicos (FECHINE; TROMPIERI, 2012). Nessa perspectiva, é possível afirmar que as temperaturas, máximas ou mínimas, são capazes de influenciar o sistema cardiovascular e favorecer a ocorrência de doenças e/ou complicações, além de contribuir para o aumento da mortalidade.

Com os resultados obtidos nesta pesquisa, verifica-se que a mortalidade por doenças cardiovasculares é influenciada pelo clima, sendo a temperatura (máxima, mínima ou média) a variável climática responsável por obter um me- lhor ajuste, visto que, à medida que a variável temperatura sofre decréscimos, o coeficiente de mortalidade se eleva. Ainda, destaca-se que, quando se utilizam as variáveis climáticas (precipitação pluvial, temperatura média e umidade relativa do ar) de forma conjunta, reunidas no modelo regressivo, são obtidos resultados ainda mais significativos, indicando assim que o estudo conjunto de variáveis atua de uma maneira mais efetiva para explicar o coeficiente de mortalidade.

Por se tratar de um estudo sobre doenças cardiovasculares, é necessário pensar na perspectiva da multifatoriedade envolvida com a mortalidade pelas DACs. Portanto, deve-se levar em consideração que o presente estudo analisou somente os números de óbitos de idosos e a influência do clima, não integrando na análise outros fatores de risco para mortalidade por DACs. A influência de fatores ambientais sobre a saúde humana é complexa e necessita de uma avaliação conjunta e interdisciplinar dos profissionais de saúde e de climatologia, dentre outros, para entender tal relação (SOUZA; SILVA; LACERDA, 2013).

\section{Conclusões}

As DACs apresentam um comportamento específico para cada faixa etária, mas, em todas as idades investigadas, o coeficiente de mortalidade evidenciou um aumento nos meses em que a atmosfera demonstra maior decréscimo de temperatura (maio, junho e julho). As variáveis climáticas exercem influên- 
cia na mortalidade por doenças cardi vasculares, destacando a influência da temperatura. Um conjunto de variáveis exercem uma maior influência nos coeficientes de mortalidade, aumentando o grau de significância e de explicação das variáveis sobre o coeficiente.

Com este estudo, verificou-se que as variáveis climáticas exerceram influência na mortalidade por doenças cardiovasculares no município de Ponta Grossa, PR, no período investigado, destacando a influência da temperatura, com fortes correlações estatísticas e elevado grau de significância.

É de grande relevância que a população idosa seja esclarecida sobre sua maior vulnerabilidade a patologias e condições climáticas, trabalhando-se, assim, a prevenção de doenças cardiovasculares com medidas individuais e coletivas de proteção à saúde e minimizando o risco do óbito evitável.

\section{Relation between variable climate and cardiovascular disease mortality}

\section{Abstract}

To evaluate the relation between mortality from cardiovascular diseases and climatic variables, as well as describe the elderly deaths in the city of Ponta Grossa, Paraná, in a period of 16 years (1998-2013). Epidemiological data were analyzed mortality rate format along with weather data by the Pearson Correlation Coefficient Test and Multiple Linear Regression. Cardiovascular diseases were the major cause of mortality in the elderly population of Ponta Grossa. The mortality rate showed a little seasonal pattern during the series. Obtained strong negative correlations with temperatures showed high statistical significance. The annual regressive models were highly significant and a good degree of fit. It is possible to prove that climate variables have influence on cardiovascular disease mortality in the city of Ponta Grossa, highlighting the influence of temperature.

Keywords: Mortality. Climatology. Epidemiology. Cardiovascular deseases. Aged.

\section{Referência}

ALVES, L. C. et al. A influência das doenças crônicas na capacidade funcional dos idosos do município de São Paulo, Brasil. Cadernos de Saúde Pública, Rio de Janeiro, v. 23, n. 8, p. 1924-1930, ago. 2007.

CABRERA, M. A. S.; ANDRADE, S. M.; WAJNGARTEN, M. Causas de mortalidade em idosos: estudo de seguimento de nove anos. Geriatria \& Gerontologia, São Paulo, v. 1, n. 1, p. 14-20, set. 2007.

DEPARTAMENTO DE INFORMÁTICA DO SISTEMA ÚNICO DE SAÚDE - DATASUS. Informações de Saúde (TABNET): Epidemiológicas e Morbidade. 2016. Disponível em: <http://www2.datasus.gov.br/DATASUS/ index.php?area=0203>. Acesso em: 15 mar. 2016.

DOWNING, D.; CLARK, J. Regressão linear múltipla. In: Estatística aplicada. 2. ed. São Paulo: Saraiva, 2003. p. 263-277.

FECHINE, B. R. A.; TROMPIERI, N. O processo de envelhecimento: as principais alterações que acontecem com o idoso com o passar dos anos. Revista Científica Internacional, Campos dos Goytacazes, v. 20, n. 1, p. 106-194, jan./mar. 2012.

GENARO, V. Relações entre o tempo atmosférico e doenças cardiorespiratórias na cidade de Cordeirópolis-SP. Dissertação (Mestrado em Geografia) - Universidade Estadual Paulista, Rio Claro, SP, 2011. 
GOTTLIEB, M. G. V. et al. Envelhecimento e longevidade no Rio Grande do Sul: um perfil histórico, étnico e de morbi-mortalidade dos idosos. Revista Brasileira de Geriatria e Gerontologia, Rio de Janeiro, v. 14, n. 2, p. 365-380, jan./mar. 2011.

JORGE, M. H. P. M. et al. A mortalidade de idosos no Brasil: a questão das causas mal definidas. Epidemiologia do Serviço de Saúde, Brasília, v. 17, n. 4, p. 271-281, out./ dez. 2008.

MIRANDA, M. J. A relação das condições meteorológicas com morbi-mortalidade na cidade de São Paulo: uma perspectiva geográfica. In: ENCONTRO DE GEÓGRAFOS DA AMÉRICA LATINA, 12, abr. 2009, Montevidéu, Uruguai. Anais... Montevidéu, Uruguai: PPGEO, 2009. p. 01-12.

NOGUEIRA, V. B. M. et al. Efeitos das alterações climáticas e antrópicas na saúde do idoso. Revista Brasileira das Ciências do Envelhecimento Humano, Passo Fundo, v. 8, n. 1, p. 88-106, jan./abr. 2011.

PASCOALINO, A.; PITTON, S. E. C. Variações atmosféricas e saúde: influências da sazonalidade e dos tipos de tempo de inverno na mortalidade por doenças cardiovasculares na cidade de Limeira/SP. ACTA Geográfica - Edição Especial Climatologia Geográfica, Boa Vista, p. 239-256, 2012.

PIUVEZAM, G. et al. Mortalidade em idosos por doenças cardiovasculares: análise comparativa de dois quinquênios. Arquivo Brasileiro de Cardiologia, São Paulo, v. 105, n. 4, p. 371-380, jul. 2015.

REDE INTERAGENCIAL DE INFORMAÇÃO PARA A SAÚDE. Fichas de qualificação de indicadores. In: . Indicadores básicos para a saúde no Brasil: conceitos e aplicações. 2. ed. Brasília: Organização Pan-Americana da Saúde, 2008. p. 130.

RUMEL, D. et al. Infarto do miocárdio e acidente vascular cerebral associados à alta temperatura e monóxido de carbono em área metropolitana do sudeste do Brasil. Revista
Saúde Pública, São Paulo, v. 27, n. 1, p. 15-22, mar. 1993.

SILVA, E. N.; RIBEIRO, H.; SANTANA, P. Clima e saúde em contextos urbanos: uma revisão da literatura. Revista Eletrónica de Geografía y Ciencias Sociales, Coimbra, v. 19 , p. 123-134, set. 2014.

SOUZA, V. C.; SILVA, S. S. F.; LACERDA, C. S. Ambiente atmosférico (clima) e a ocorrência da insuficiência coronariana em Campina Grande-PB. In: CONGRESSO BRASILEIRO DE GESTÃO AMBIENTAL E SUSTENTABILIDADE, 1, 2013, João Pessoa-PB. Anais... João Pessoa, 2013. p. 590-592.

VERAS, R. P. Estratégias para o enfrentamento das doenças crônicas: um modelo em que todos ganham. Revista Brasileira de Geriatria e Gerontologia, Rio de Janeiro, v. 14, n. 4, p. 779-786, jan. 2011.

VERAS, R. P. Prevenção de doenças em idosos: os equívocos dos atuais modelos. Cadernos de Saúde Pública, Rio de Janeiro, v. 28, n. 10 , p. 1834-1840, out. 2012. 